\title{
Evidence of Pulsars Metamorphism and Their Connection to Stellar Black Holes
}

\author{
Ahmad A. Hujeirat \\ IWR, Universität Heidelberg, Heidelberg, Germany \\ Email:AHujeirat@uni-hd.de
}

How to cite this paper: Hujeirat, A.A. (2018) Evidence of Pulsars Metamorphism and Their Connection to Stellar Black Holes. Journal of Modern Physics, 9, 532-553.

https://doi.org/10.4236/jmp.2018.94037

Received: February 2, 2018

Accepted: March 11, 2018

Published: March 14, 2018

Copyright $\odot 2018$ by author and Scientific Research Publishing Inc. This work is licensed under the Creative Commons Attribution International License (CC BY 4.0).

http://creativecommons.org/licenses/by/4.0/

c) (i) Open Access

\section{Abstract \\ It is agreed that the progenitors of neutron stars (-NSs) and black holes} (-BHs) should be massive stars with $\mathrm{M} \geq 7 M_{\odot}$. Yet none of these objects have ever been found with $\left[2 M_{\odot} \leq \mathrm{M} \leq 4 M_{\odot}\right]$. Moreover, numerical modelings show that NSs of reasonable masses can be obtained only if the corresponding central density is beyond the nuclear one: an unverifiable density-regime with unknown physics. Here I intend to clarify the reasons underlying the existence of this mass-gap and propose a new class of invisible ultra-compact objects: the end-stage in the cosmological evolution of pulsars and neutron stars in an ever expanding universe. The present study relies on theoretical and experimental considerations as well as on solution of the non-linear TOV equation modified to include a universal scalar field $-\phi$ at the background of supranuclear densities. The computer-code is based on finite volume method using both the first-order Euler and fourth-order Rugge-Kutta integration methods. The inclusion of $\phi$ at zero-temperature is motivated by recent observations of the short-living pentaquarks at the LHC. Based on these studies, I argue that pulsars must be born with embryonic super-baryons (SBs) that form through merger of individual neutrons at their centers. The cores of SBs are made of purely incompressible superconducting gluon-quark superfluids (henceforth SuSu-fluids). Such quantum fluids have a uniform supranuclear density and governed by the critical EOSs for baryonic matter $P_{b}=\mathcal{E}_{b}$ and for $\phi$-induced dark energy $P_{\phi}=-\mathcal{E}_{\phi}$. The incompressibility here ensures that particles communicate at the shortest possible time scale, superfluidity and superconductivity enforce SBs to spin-down promptly as dictated by the Onsager-Feynman equation and to expel vortices and magnetic flux tubes, whereas their lowest energy state grants SBs lifetimes that are comparable to those of protons. These extra-ordinary long lifetimes suggest that conglomeration of SuSu-objects would evolve over several big bang events to possibly form dark matter halos that embed the galaxies in the observable universe. Pulsars and young neutron 
stars should metamorphose into SuSu-objects: a procedure which is predicted to last for one Gyr or even shorter, depending on their initial compactness. Once the process is completed, then they become extraordinary compact and turn invisible. It turns out that recent observations of particle collisions at the LHC and RHIC, observations of glitching pulsars and primordial galaxies remarkably support the present scenario.

\section{Keywords}

Relativity, General, Black Hole Physics, Pulsars, Neutron Stars, Superfluidity, Low Temperature and Condensed Matter, QCD, Dark Energy, Dark Matter

\section{Introduction}

Pulsars and NSs are considered to be made of superfluids governed by triangular lattice of quantized vortices as prescribed by the Onsager-Feynman equation: $\oint \boldsymbol{v} \cdot \mathrm{d} \boldsymbol{l}=\frac{2 \pi \hbar}{m} N \cdot \boldsymbol{v}, \mathrm{d} \boldsymbol{l}, \hbar, m$ here denote the velocity field, the vector of

line-element, the reduced Planck constant and the mass of the superfluid particle pair, respectively.

Accordingly, Crab pulsar should have approximately $N_{n}=8.6 \times 10^{17}$ neutron and $N_{p} \approx 10^{30}$ proton-vortices (Figure 1 ).

Let the evolution of the number density of vortex lines, $n_{v}$, obey the following advection-diffusion equation:

$$
\frac{\partial n_{v}}{\partial t}+\nabla \cdot n_{v} \boldsymbol{u}_{f}=v_{t} \Delta n_{v}
$$

where $t, \boldsymbol{u}_{f}, v_{t}$ denote the transport velocity at the cylindrical radius $r=r_{f}$ and dissipative coefficient in the local frame of reference, respectively. When $v_{t}=0$, then the radial component of $\boldsymbol{u}_{f}$ in cylindrical coordinates reads: $u_{f}^{\max } \approx-(\dot{\Omega} / \Omega) r>0$. In the case of the Crab, this would imply that approximately $10^{6}$ neutron vortices must be expulsed/annihilated each second, and therefore the object should switch off after $10^{6}$ or $10^{13} \mathrm{yr}$, depending on the underlying mechanism of heat transport (see [1] [2], and the references therein). However, both scenarios are contrary to observation, as numerous NSs have been found, which are older than $10^{6} \mathrm{yrs}$, though non of them is older than $10^{9} \mathrm{yrs}$. On the other hand, recent numerical calculations of superfluids reveal generation of large amplitude Kelvin waves that turn superfluids turbulent (see [3] [4] [5], and the references therein). It is therefore unlikely that trillions of Kilometer-long neutron and protons-vortices inside pulsars and NSs would behave differently. In this case, $u_{f}$ should be replaced by a mean turbulent velocity $\left\langle u_{f}\right\rangle^{t}$ with $u_{f}^{\max }$ being an upper limit ${ }^{1}$. As the number of vortex lines decreases with

${ }^{1}$ The rotational energy associated with the outward-transported vortex lines from the central regions is turbulently re-distributed in the outer shells and should not necessarily suffer a complete annihilation. 


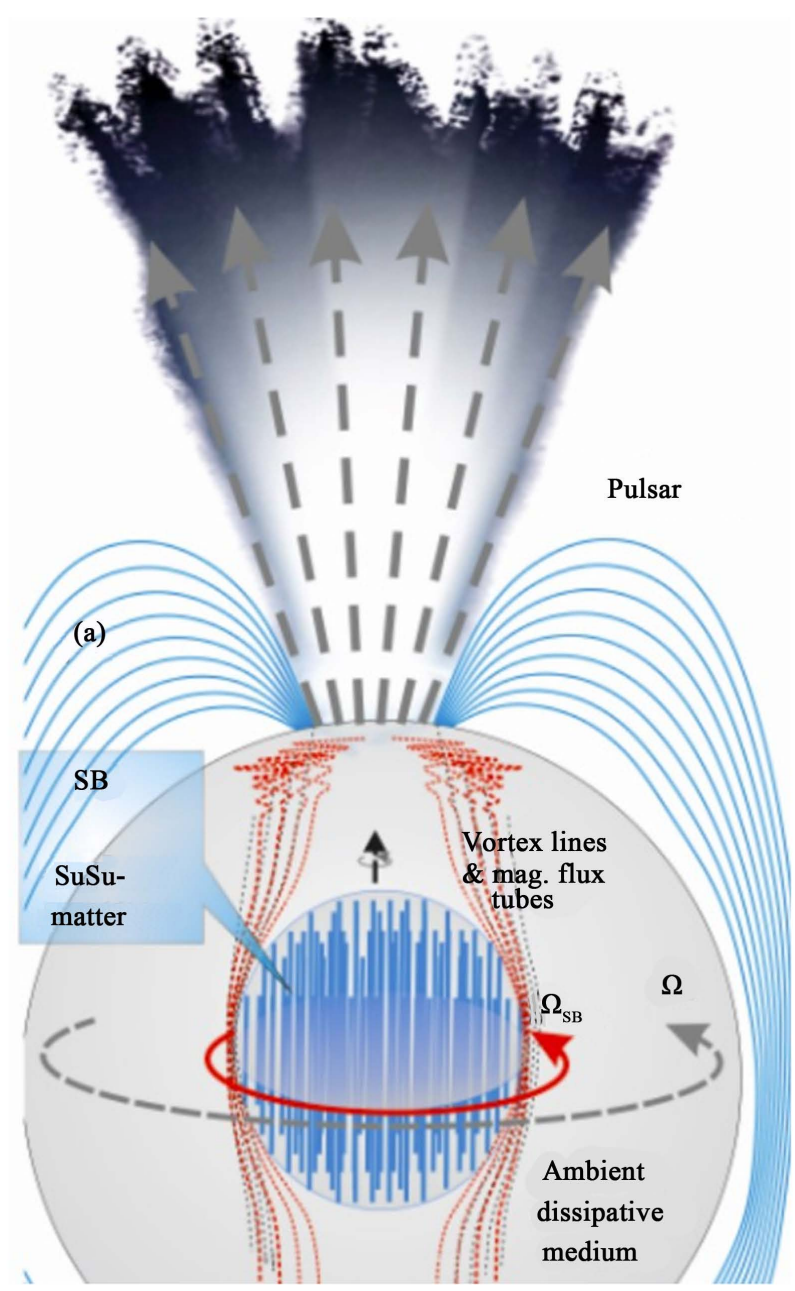

Figure 1. A magnetized pulsar is born with an embryonic rigid-body rotating super-baryon $(\mathrm{SB})$ at its center, which is made of incompressible gluon-quark superfluid. By interacting with the ambient medium, the SB expels certain number of vortex lines that are absorbed by the surrounding dissipative medium, thereby causing to promptly spin up: the exact quantum signatures of the glitches observed in pulsars and young neutron star systems.

time due to emission of magnetic dipole radiation and therefore the separation between them increases non-linearly. It is reasonable to associate a time-dependent turbulent length scale $\ell_{t}(t)$, which covers the two limiting cases: $\ell_{t}(t=0)=\ell_{0} \approx 10^{-3} \mathrm{~cm}$ and $\ell_{t}(t=\infty)=\ell_{\infty}=R_{\star}$. This yields the geometrical mean $\left\langle\ell_{t}\right\rangle=\sqrt{\ell_{0} \ell_{\infty}} \approx \mathcal{O}(10) \mathrm{cm}$. Putting terms together and using $v_{\text {tur }}=\left\langle\ell_{t}\right\rangle\left\langle u_{f}\right\rangle^{t}$ to describe the effective turbulent viscosity, we obtain an upper limit for the global diffusion time scale: $\tau_{\text {diff }}=R_{N S}^{2} / v_{\text {tur }}=\mathcal{O}\left(10^{9}\right) \mathrm{yr}$. Similarly, a comparable time scale for the Ohmic diffusion in this turbulent medium can be constructed as well. This is in line with observations, which reveal that most isolated luminous NSs known are younger than $10^{9} \mathrm{yr}$ (see [6], and the references therein). 
On the other hand, as stable degenerate NSs require the density gradient to be negative, then the very central region would be the first to be evacuated from vortex lines and all other removable energies that do not contribute significantly to the pressure. Let $r_{f}$ be the radius of the central region. The star cools and loses energy, $r_{f}$ would creep outwards with an average velocity: $\dot{r}_{f} \sim R_{\star} / \tau_{\text {diff }} \approx 10^{-10} \mathrm{~cm} / \mathrm{s}$. As I show in the next sections, the nuclear matter inside $r_{f}$ would undergo a transition from compressible dissipative neutron fluid into an incompressible gluon-quark superfluid phase; the lowest possible energy state. Once $r_{f}=R_{\star}-\epsilon \quad(\epsilon \ll 1)$, then the object turns invisible.

In analogy with normal massive luminous stars, we expect massive NSs to also switch-off earlier than their less massive counterparts. The reason is that most models of EOSs for NSs predict a correlation of $M_{N S} \propto \alpha_{s}$, where $\alpha_{s}\left(\doteq \frac{R_{S}}{R_{\star}}\right)$ denotes the compactness parameter, $R_{\star}$ and $R_{S}$ are the actual radius of the star and the corresponding Schwarzschild radius [7]. In the extremal case however, when $\alpha_{s}=1$, it is reasonable to expect that there will be no turbulence to dissipate. In this case the expression of for the global turbulent diffusion time scale should be modified as follows: $\tau_{\text {diff }}=R_{N S}^{2} / v_{\text {tur }} \rightarrow\left(R_{N S}-R_{S}\right)^{2} / v_{\text {tur }}$. In terms of NS-parameters, the turbulent viscosity reads: $v_{\text {tur }}=R_{N S}^{2}(\dot{\Omega} / \Omega) \sqrt{\left(\ell_{0} / R_{N S}\right)}$. Putting terms together, we obtain $\tau_{\text {diff }}=\left(1-\alpha_{s}\right)^{2} \sqrt{\left(R_{N S} / \ell_{0}\right)}(\Omega / \dot{\Omega})$. For a Vela-type pulsar with $\alpha_{s} \approx 2 / 3$, the effect of compactness would shorten $\tau_{\text {diff }}$ by almost one order of magnitude.

\section{The Incompressibility of Supranuclear Dense Fluids}

Modeling the internal structure of cold NSs while constraining their masses and radii to observations, would require their central densities to inevitably be much higher than the nuclear density $-\rho_{0}$ : a density regime in which all EOSs become rather uncertain and mostly acausal (see [8] [9], and the references therein). On the other hand, at very high densities, almost all EOSs converge to the stiffest EOS: $P_{\text {local }}=\mathcal{E}$ [10], where fluids become purely incompressible.

The corresponding chemical potential here reads:

$\mu=\frac{\partial \mathcal{E}_{b}}{\partial n}=\frac{P_{\text {local }}+\mathcal{E}_{b}}{n}=\frac{2 \mathcal{E}_{b}}{n}$, whose solution is:

$$
\mathcal{E}_{b}=a_{\infty} n^{2}
$$

Particles obeying this EOS communicate with the speed of the light. This implies that the number density must be upper-bounded by $n_{c r}$, beyond which local thermodynamical quantities become constants, specifically $\mu=E_{\phi}=P_{\text {local }}=$ const. In this case calculating the degenerate pressure from the local quantities becomes invalid as it yields a vanishing local pressure $\left(P_{\text {local }}=n^{2} \frac{\partial}{\partial n}\left(\mathcal{E}_{b} / n\right)=0\right)$.

Even if the fluid were weakly-compressible only, $\nabla P_{\text {local }}$ would be too 
smooth to hold the object gainst its own self-gravity. This becomes even blatant at the center, where the gradient of the pressure vanishes to meet the regularity condition. The usual adopted strategy to escape this pressure-deficiency is to enforce an unfounded inward-increase of $\mathcal{E}_{b}$ as $r \rightarrow 0$, resulting therefore in unreasonably large central densities.

Alternatively, one could argue that the strong gravitational field would enforce fusing of neutrons at the center of NSs and form a sea of gluon-quark fluid. However, it was argued that a phase transition from normal nuclear matter into a gluon-quark plasma (GQP) under classical conditions is unlikely [8] [11]. However, it was found by that their analysis was incorrect [12]. The GQPs in these studies were assumed to be locally conformal, compressible and governed by a non-local and constant bag pressure.

However, the situation may differ if there is a mechanism at the background of supranuclear densities, that among others, is capable of injecting energy into the system. This in turn would effectively increase the mass of the object, steepen the curvature of the embedding spacetime and compress the nuclear fluid up to the saturation level, beyond which it becomes purely incompressible. The underlying conjecture here is that compression would mainly affect the distance between individual neutrons, $\ell_{n}$, rather than the distance, $\ell_{q}$, between individual quarks inside single neutrons (Figure 2 und Figure 3).

In fact there are at least two observational facts that in favor of this argument:

1) Massless gluons mediate the strong force between quarks with the speed of light, whereas global compression of the central fluid-core proceeds almost in a quasi-stationary manner. Hence, as the quark contribution to baryon mass is

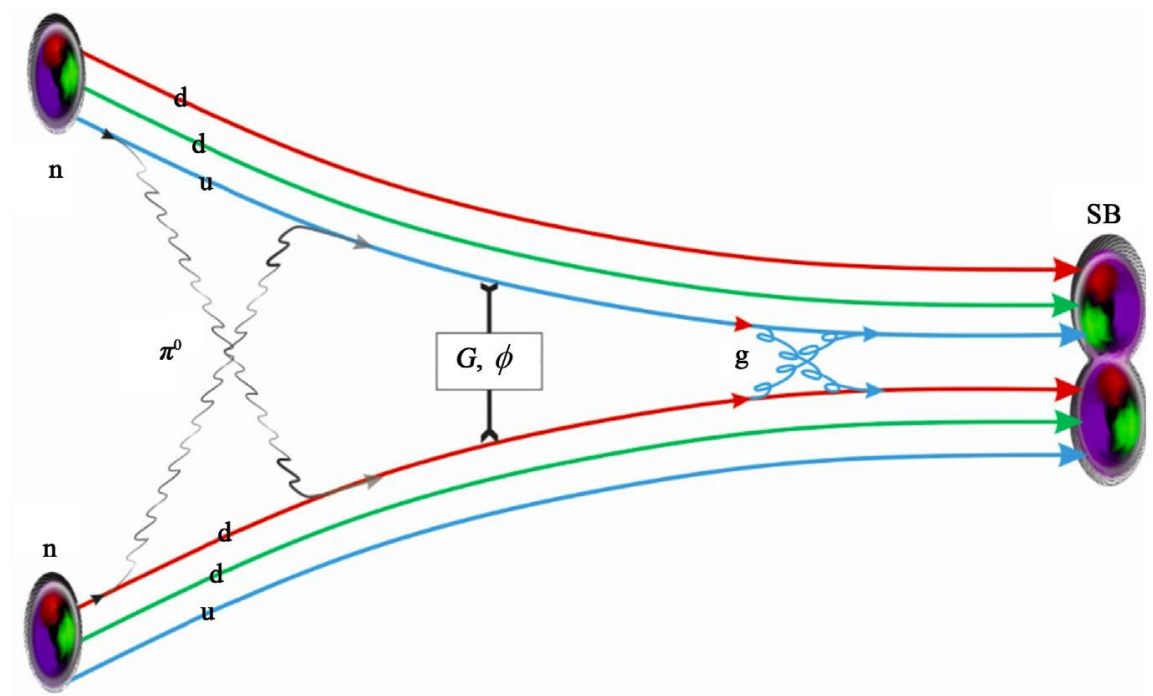

Figure 2. A Feynmann-like diagram of two interacting neutrons at the center of a pulsar (time runs from left-to-right). These two neutrons, that are made of two "d" and "u" quarks approach each other under the effect of the gravitational and the scalar fields. Once the distance between them falls below a certain critical value, the residual strong force undergo a "gluonization" procedure necessary for efficiently mediating the strong force between quarks. Here the two particles merge together and form a super-baryon. 


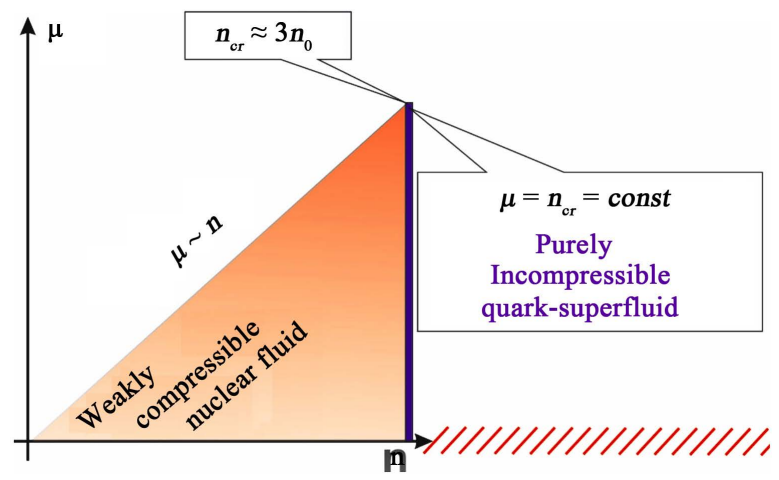

Figure 3. A schematic description of the chemical potential $\mu, \mu^{\prime}(\doteq \partial \mu / \partial n)$ versus the number density $n$ at the centers of NSs. At a critical central density, $n_{c r}$, the universal scalar field $\phi$ is set to provoke a crossover phase transition from compressible and dissipative neutron fluid into incompressible GQ-superfluid. The transition front creeps from inside-to-outside to reach the surface of the object on the scale of one Gyr.

negligibly small, compressibility of the gluon-quark plasma would violate the causality principle.

2) The quark potential $\mathcal{V}_{q}$ inside baryons increases with the radius to attains maximum at the boundaries $-r_{b}$. Quark confinement however requires that $\mathcal{V}_{q}\left(r_{b}\right) \approx 0.94 \mathrm{GeV}$. In this case the effective/reactive velocity at the boundary would read: $\langle V\rangle=\Delta x / \Delta t=\Delta x \Delta E / h \approx c$, where we set $\Delta x=r_{b}=10^{-13} \mathrm{~cm}$. Consequently, the reaction time of GQPs inside neutrons to whatsoever external compressional effects is of order $10^{-24} \mathrm{sec}$ : the shortest possible time scale in the entire system.

Moreover, recalling that the lifetime of neutrons in free space is extremely short compared to that of protons, then compression of GQPs inside neutrons most likely would provoke a runaway decay into protons and would give rise to neutrino-dominated electromagnetic eruptions. However, such events can be safely ruled out by observations.

3) Recent RHIC and LHC experiments have revealed that the matter resulting from smashed protons behaves like fluids, whose constituents move collectively and relatively slowly. These observations are in line with the basic properties of incompressible fluids rather than randomly moving particles in plasmas.

Moreover, the coupling constant, $\breve{\alpha}_{S}$, in QCD is inversely proportional to the density: $\breve{\alpha}_{S} \sim 1 / \rho$. Hence matter with $\rho>\rho_{0}$ and $T=0$ yields automatically $\breve{\alpha}_{S} \ll 1$. This means that the interaction strength is at lowest and therefore the quarks must be moving freely, practically unaffected by whatsoever external forces.

Consequently, as GQPs inside neutrons are incompressible, we expect nuclear fluid inside SBs to be indifferent. This implies that, when the distance between neutrons becomes critically small, e.g. $\ell_{n} / \ell_{q}=O(1)$, then the pions, $\pi^{0}$, the 
carriers of the residual strong force between neutrons, become sufficiently energetic and overcome the repulsive barrier, where they go through a "gluonization" process, which subsequently enables them to mediate the strong force between quarks inside SBs efficiently (Figure 2).

Similar to classical incompressible normal fluids, the dynamics of the fluid is governed by a non-local pressure whereas the local pressure acts a source for perturbations only. For constructing the non-local pressure an ad hoc non-local scalar field in introduced [13].

\section{The Onset of Superfluidity}

In the presence of magnetic torque and in the absence of energy generation at the center of pulsars, all types of energies, including magnetic, rotational and rest of thermal energies must diffuse outwards. This energy loss enforces the degenerate core to cool down to $T=0$ and to increase its density. In this case the corresponding de Broglie wave length, $\left(\lambda_{D e} \propto 1 / T\right)$, which coincides with the radius of the core, increases and the enclosed particles start occupying the same quantum state, hence entering the superfluid phase with vanishing entropy: $\left(S=k_{B} \ln (\Omega)=0\right)$. In fact this is the lowest energy state possible in the system, which is termed here $L$-State.

Indeed, recent RHIC and LHC-experiments reveal that GQPs inside smashed protons, which are generally at much higher energy states than the states considered here, were found to be nearly perfect with even smaller shear viscosity $(\eta)$ over entropy $(s)$ ratio [14] [15]. Taking into account that both:

$$
\lim _{T \rightarrow 0} \eta=0 \text { and } \lim _{T \rightarrow 0} s=0
$$

and that $\eta / s \propto T$, we conclude that:

$$
\lim _{T \rightarrow 0}\left(\frac{\eta}{s}\right)=\lim _{T \rightarrow 0}\left(\frac{\partial \eta / \partial T}{\partial s / \partial T}\right) \sim T^{\Gamma},
$$

where $\Gamma$ a constant exponent.

This implies that the shear viscosity is much more sensitive to the temperature and converges more quickly to zero than entropy. Hence, we predict the interiors of SBs, which result from fusion of individual neutrons in the $L$-State, to be in a purely superfluid and superconducting phase.

The injected dark energy by $\phi$ may affect the central nuclear fluid in two way: it first strengthens the curvature of the embedding spacetime, enhancing compression of the central fluid, and secondly, it provokes the "gluonization" procedure:

$$
\Delta E_{\phi}+\Delta E_{G}+\pi^{0} \rightarrow \Delta E_{g}^{S B},
$$

where $\Delta E_{\phi}, \Delta E_{G}, \pi^{0}, \Delta E_{g}^{S B}$ denote the local energy enhancement by $\phi$ and " $G$ ' ( $\doteq$ gravitational force), the pion energy and the resulting excess of energy gained by the SB, respectively. This reaction is assumed to proceed directly and silently without destroying the superfluidity character of GQ-fluid inside SBs. 
On the other hand, the rotational torque of the dissipative ambient medium exerted on the SBs would enforce them co-rotate uniformly. However, SBs are quantum identities and therefore must obey the laws of quantum mechanics. This means that their dimensions and energies can accept discrete values only. It turns out that SBs evolve in a discrete manner as prescribed by Onsager-Feymann equation in superfluids. SBs here must eject a certain number of vortex lines in order to increase their dimensions [16]. Following this scenario, the internal structure of pulsars and UCOs looks as follow:

1) Pulsars are born with SB-embryos at their centers, which are made of an incompressible superconducting gluon-quark superfluid,

2) a dissipative neutron fluid that surrounds the $S B$, and

3) a geometrically thin boundary layer in-between (BL), where the residual of the strong nuclear force becomes dominant over the viscous forces. The viscosity of the neutron fluid here decreases strongly inwards and vanishes at the boundary of the SB. The BL here is practically the zone, where the neutron fluid is prepared to match the physical conditions governing GQ-fluids inside SBs prior to their merger with other neutrons. It turns out that these merger events are identical to the sudden glitch phenomena observed in pulsars and young neutron star systems [16]. Accordingly, once the rotational frequency of the ambient medium falls below a certain critical frequency $\Omega_{c}$, the SB then undergo a sudden spin-down to the next quantum-allowable frequency, thereby expelling a certain number of vortices, which are then absorbed by the ambient medium and causes the observed spin-up of pulsars (see Figure 4). However, the excess of rotational energy is then viscously-redistributed into the entire ambient dissipative medium: a process that may last for weeks or even months.

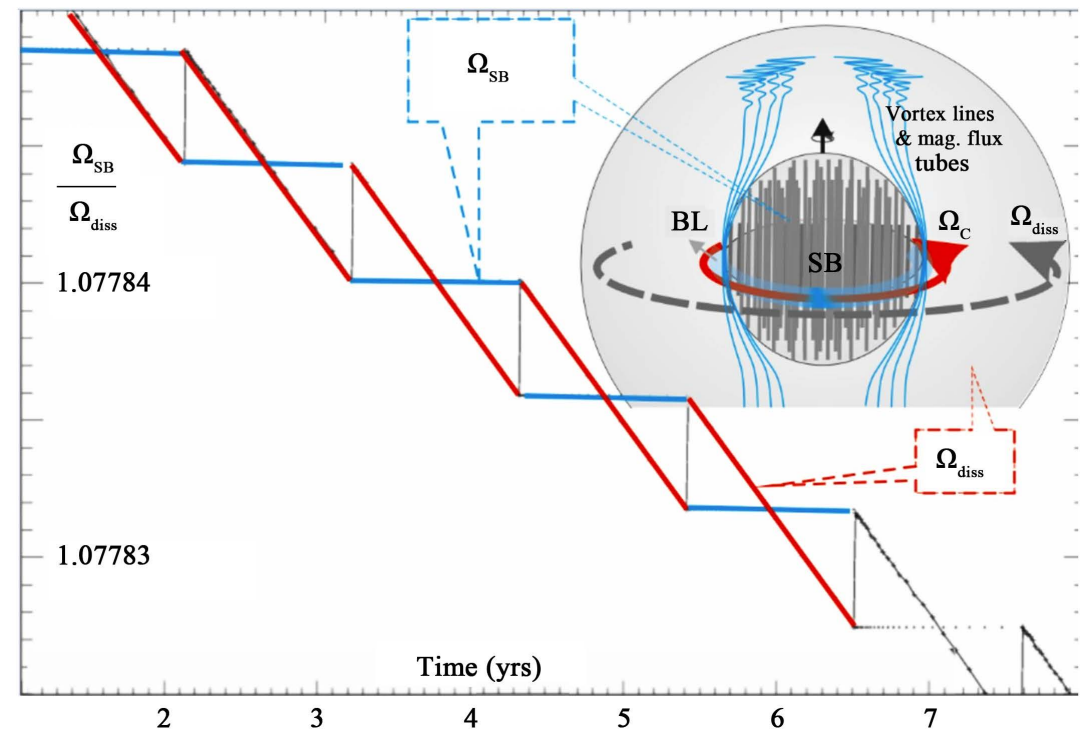

Figure 4. The discrete time-evolution of the rotational frequency of an $\mathrm{SB}\left(\Omega_{\mathrm{SB}} /\right.$ blue colour) and of the ambient dissipative medium $\left(\Omega_{\text {diss }}\right.$ /red colour). The rotational frequency and the dimension of the SB evolve as dictated by the Onsager-Feynmann equation for superfluids (Hujeirat 2017c). 
Indeed, very recent observations appear to confirm our scenario. [17] [18] found that the mean fractional moment of inertia in the glitching pulsars correlates weakly with the pulsar spin, implying therefore that glitches are provoked by a central core of different physical properties. A obvious evidence therefor has been found the accretion-powered pulsar SXP 1062 [19]. Obviously, although the source was subject to a short X-ray outburst that went off 25 days prior to the glitch event, the event did not alter the spin-down of the source. This indicates that glitches are triggered by the core of the NS rather than by the outer shells.

\section{Crossover Phase Transition}

For $\rho>\rho_{0}$, short-range interactions between particles mediated by the exchange of vector mesons most likely would enhance the convergence of the EOSs towards $P \rightarrow \mathcal{E}_{b} \sim n_{b}^{2}$ (see [7] [10] [20], and the references therein). The chemical potential here: $\mu\left(\doteq\left(\mathcal{E}_{b}+p\right) / n_{b}\right)$ increases linearly with the number density $n_{b}$. Matter with $\mathrm{d} \mu / \mathrm{d} n>0$ is classified here as H-State and depicted in red-color in Figure 3.

However in the absence of energy generation, degenerate matter cannot hold a correlation of the type $\mu \sim n_{b}$ indefinitely and it must terminate at a certain critical density $n_{c r}$. This agrees with the two facts: 1) central densities in NSs, $\rho_{c}$, increase with their masses and 2) $\rho_{c}$ must be upper-bounded by $\rho_{c} \leq 12.5 \times \rho_{0}$ in order to fit the observed mass function (see [21], and the references therein).

On the other hand, in an ever expanding universe, the eternal-state of matter should be the one at which the internal energy attains a global minimum in spacetime (zero-temperature, zero-entropy and where Gibbs energy per baryon is lowest, i.e. in the L-State). Taking into account that $\mu(r) \mathrm{e}^{\mathcal{V}(r)}=$ const inside massive NSs together with the a posteriori result that $\mathrm{e}^{\mathcal{v}} \ll 1$ we conclude that $\mu \sim \mathcal{E}_{b} / n_{b}=$ const. Under these conditions the gradient of the local pressure $P_{\text {local }}$ vanishes and a non-local pressure $P_{N L}$ is necessary to avoid self-collapse of the object into a $\mathrm{BH}$.

${ }^{2}$ The slop of the chemical potential $\mathrm{d} \mu / \mathrm{d} n$ between the $\mathrm{H}$ and L-states in the $\mathrm{n}$-space could attain positive, negative or even discontinuous values.

However the case with $\mathrm{d} \mu / \mathrm{d} n>0$ should be excluded, as it implies that the eternal state of matter would be more energetic than the H-State, which is a contradiction by construction. Similarly, the case $\mathrm{d} \mu / \mathrm{d} n<0$ is forbidden as it would violate energy conservation (; $\mathrm{d} \mu / \mathrm{d} n<0 \Leftrightarrow \mathrm{d} P / \mathrm{d} n<0 \Leftrightarrow$ adding more particles yields a smaller pressure). Moreover, let us re-write the TOV equation in terms of $\mu$ :

$$
\frac{\mathrm{d} \mu}{\mathrm{d} r}=-\frac{G}{c^{2} r^{2}}\left(\frac{\mathrm{d} \mathcal{E}_{b}}{\mathrm{~d} \mu}\right)\left(\frac{\mathrm{d} \mu}{\mathrm{d} n}\right)\left(\frac{m+4 \pi r^{3} P}{1-r_{\mathrm{s}} / r}\right) .
$$

${ }^{2}$ Classically incompressible fluids with $E=$ const have negative local pressure. Therefore an acausal non-local pressure is usually used for stabilizing the fluid configurations modelled by the incompressible Navier-Stokes equations. 
Obviously, as $\mu>0$, a negative $\mathrm{d} \mu / \mathrm{d} n$ would destabilize the hydrostatic equilibrium, unless external sources are included, e.g. bag energy and/or external fields.

Therefore, although a first order phase transition may not be completely excluded, a crossover phase transition into an incompressible superfluid phase with $\mu=\mu\left(n=n_{c r}\right)=$ const would be more likely. Here, $\mu$ and $P$ on both sides of the transition front are equal and, with the help of an external field, both $\left(\mathcal{E}_{\text {tot }} / n\right)^{+}$and $\left(\mathcal{E}_{\text {tot }} / n\right)^{-}$across the front can be made continuous (Figure 3 ).

In the present study, the crossover phase transition corresponds to "silent" mergers of baryons forming SBs. Inside SBs, the fluids have $\mu=n=$ const and $T=0$ and therefore vanishing entropy. To overcome the repulsive barrier between individual neutrons and provoke their merger, external forces are required. In the present study, we assume that, in addition to compression by self-gravity, there is a universal scalar field, $\phi$, at the background of supranuclear dense matter, which provide the energy required for forming an eternal stable GQ-cloud inside SBs. In this case, the dark energy potential associated with $\phi$ should have similar effects as the generalized quark-potentials inside individual neutrons, namely:

$$
\mathcal{V}_{\phi}=a_{\phi} r^{\gamma}+b_{\phi}+\frac{c_{\phi}^{E M}}{r}
$$

where $a_{\phi}, b_{\phi}, c_{\phi}^{E M}$ are constant coefficients (see [22], and the references therein). Without loss of generality, we may set $c_{\phi}^{E M}=0$ and $\gamma=2$. As the scalar field is universal, its special and temporal variations are assumed to vanish. Therefore, the expression $\mathcal{E}_{\phi}=\frac{1}{2} \dot{\phi}^{2}+V(\phi)+\frac{1}{6}(\nabla \phi)^{2}$ reduces to $\mathcal{E}_{\phi}=V(\phi)=\mathcal{V}_{\phi}$. By requiring the chemical potential at $r=0$ to be upper-limited by the deconfinement energy of quarks inside a single neutron, then a Gibbs function of the following type can be constructed:

$$
f(n)=\frac{\mathcal{E}_{b}+\mathcal{E}_{\phi}}{n}-0.939 \mathrm{GeV} .
$$

Using the scalings $[\rho]=10^{15} \mathrm{~g} / \mathrm{cm}^{3}\left(\doteq 0.597 / \mathrm{fm}^{3}\right)$, chemical potential (energy per particle) $[\mu]=1 \mathrm{GeV}$, we then obtain $\left[a_{\infty}\right]=1.674 \mathrm{GeV} \cdot \mathrm{fm}^{3}$ and $\left[a_{\phi}\right]=5.97 \times 10^{-39} \mathrm{GeV} / \mathrm{fm}^{5}$ and $\left[b_{\phi}\right]=0.597 \mathrm{GeV} / \mathrm{fm}^{3}$.

Substituting $\mathcal{E}_{b}=a_{0} n^{2}$ and $V_{\phi}(r)$ in Equation (5) at $r=0$, then $f(n)$ reduces to:

$$
f(n)=a_{\infty} n+\frac{b_{\phi}}{n}-0.9396 .
$$

The Gibbs function here may accept several minima at $n_{\min }=\left(b_{\phi} / a_{\infty}\right)^{1 / 2}$, though $f\left(n=n_{\min }\right)$ doesn't necessary vanish. However $f(n)>0$ and $f(n)<0$ should be excluded, as they are energetically not suited for a smooth crossover phase transitions.

On the other hand, by varying $a_{\infty}$ and $b_{\phi}$, a set of minima could be found. 
One way to constrain $b_{\phi}$ is to relate it to the canonical energy scale characterizing the effective coupling of quarks, i.e. $b_{\phi}=0.221$ (see [23], and the references therein). Indeed, as shown in Figure 5, $f(n)$ attains a zero-minimum at $n \approx 3 n_{0}$ for $a_{\infty}=1.0$.

\section{The Governing Equations and the Solution Procedure}

The investigation here is based on numerical solving the TOV equation modified to include scalar fields $(\phi)$. The modified stress energy tensor reads:

$$
T_{\mu v}^{\text {mod }}=T_{\mu v}^{0}+T_{\mu v}^{\phi} .
$$

The superscripts " 0 " and " $\phi$ ” correspond to baryonic and scalar field tensors:

$$
\begin{aligned}
& T_{\mu \nu}^{0}=-P^{0} g_{\mu \nu}+\left(P^{0}+E^{0}\right) U_{\mu} U_{v} \\
& \text { and } T_{\mu \nu}^{\phi}=\left(\partial_{\mu} \phi\right)\left(\partial_{\nu} \phi\right)-g_{\mu \nu}\left[\frac{1}{2}\left(\partial_{\sigma} \phi\right)\left(\partial^{\sigma} \phi\right)-V(\phi)\right] .
\end{aligned}
$$

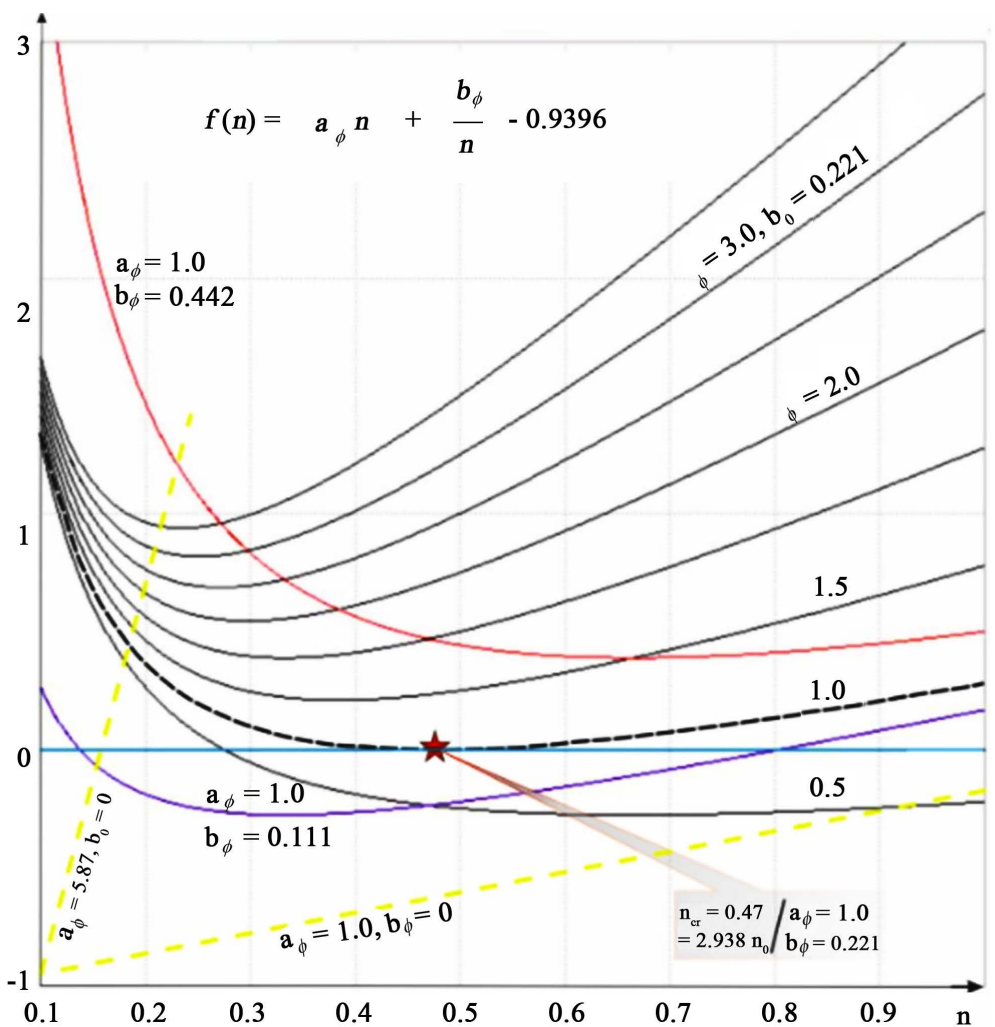

Figure 5. The modified Gibbs function $f(n)$ versus baryonic number density $n$ (in units of $n_{0}$ ) is shown for various values of $a_{\infty}$ and $b_{\phi}$. Obviously, $a_{\infty}=1$ and $b_{\phi}=0.221$ appear to be the most appropriate parameters compatible with QCD. The value $b_{\phi}=0.221$ corresponds to the canonical energy scale characterizing the effective coupling of quarks inside individual hadrons. The Gibbs function $f(n)$ here attains a zero-minimum at $n=2.938 n_{0}$, at which $\phi$ is set to provoke a phase transition into the SuSu-fluid state. 
$U_{\mu}$ here is the 4-velocity, the subindices $\mu, v$ run from 0 to 3 and $g_{\mu v}$ is a background metric of the form:

$$
g_{\mu \nu}=\mathrm{e}^{2 \nu} \mathrm{d} t^{2}-\mathrm{e}^{2 \lambda} \mathrm{d} r^{2}-r^{2} \mathrm{~d} \theta^{2}-r^{2} \sin ^{2} \theta \mathrm{d}^{2} \varphi^{2},
$$

where $\mathcal{V}, \lambda$ are functions of the radius.

Assuming the configuration to be in hydrostatic equilibrium, then the GR field equations, $G_{\mu \nu}=-8 \pi G T_{\mu \nu}$ reduce into the generalized TOV equations:

$$
\frac{\mathrm{d} P}{\mathrm{~d} r}=-\frac{G}{c^{4} r^{2}}[\mathcal{E}+P]\left[m(r)+4 \pi r^{3} P\right] /\left(1-r_{s} / r\right),
$$

where $m(r)=4 \pi \int \mathcal{E} r^{2} \mathrm{~d} r$ is the total enclosed mass: $\mathcal{E}=\mathcal{E}^{0}+\mathcal{E}^{\phi}, \quad P=P^{0}+P^{\phi}$, where $\mathcal{E}^{\phi}=\frac{1}{2} \dot{\phi}^{2}+V(\phi)+\frac{1}{2}(\nabla \phi)^{2}, \quad P^{\phi}=\frac{1}{2} \dot{\phi}^{2}-V(\phi)-\frac{1}{6}(\nabla \phi)^{2}$.

$V(\phi)$ here denotes the interaction potential of the scalar field with the baryonic matter, i.e., the rate at which dark energy is injected into the system and $\dot{\phi}$ is the time-derivative of $\phi$.

Our reference object is a NS with $1.44 \mathcal{M}_{\odot}$, a radius $R_{\star}=2 \times R_{S}$, where $R_{S}$ is the Schwarzschild radius. The universal scalar field, $\phi$ is assumed to be spatially and temporarily constant, whereas $V_{\phi}$ is taken from Equation (4). The non-dimensional value of $a_{\phi}$ is chosen to fulfill the a posteriori requirement: $R_{\star}=R_{S}+\epsilon$, where $0 \leq \epsilon \ll 1$. In most of the cases considered here, $b_{\phi}$ is set to be identical to the canonical energy scale at which momentum transfer between quarks saturates, i.e., $b_{\phi}=0.221 \mathrm{GeV}$. The fluid in the post transition phase is governed by the EOS: $P^{0}=P_{L}=\mathcal{E}^{0}=\rho_{c r} c^{2}=$ const .

For a given central density, the solution procedure adopted here is based on integrating the TOV-equations for the pressure, enclosed mass and for the pseudo-gravitational potential from inside-to-outside, using either the first order Euler or the fourth order Runge-Kutte integration methods.

For verification purposes both integration methods have been employed using a polytropic EOS (Figure 6).

In the incompressible case, i.e. $(\mathcal{E}=$ const $)$, the spatial variation of the local pressure diverges completely and becomes unphysical. Unlike EOSs in compressible normal plasmas, EOSs in incompressible superfluids are non-local. Here constructing a communicator that merely depends on local exchange of information generally would not be sufficient for efficiently coupling different/remote parts of the fluid in a physically consistent manner. In the interior of neutron stars, the local pressure depends, not only on the global compactness of the object, but it becomes even acausal whenever the global compactness is enhanced.

This is similar to the case when solving the incompressible Navier-Stokes equations, where an additional Laplacian for describing the spatial variation of a non-local scalar field is constructed to generate a pseudo-pressure (actually a Lagrangian multiplier), which, again, does not respect causality (Figure 7).

Similarly, in Figure 8 and Figure 9/top we show the profiles of the negative 


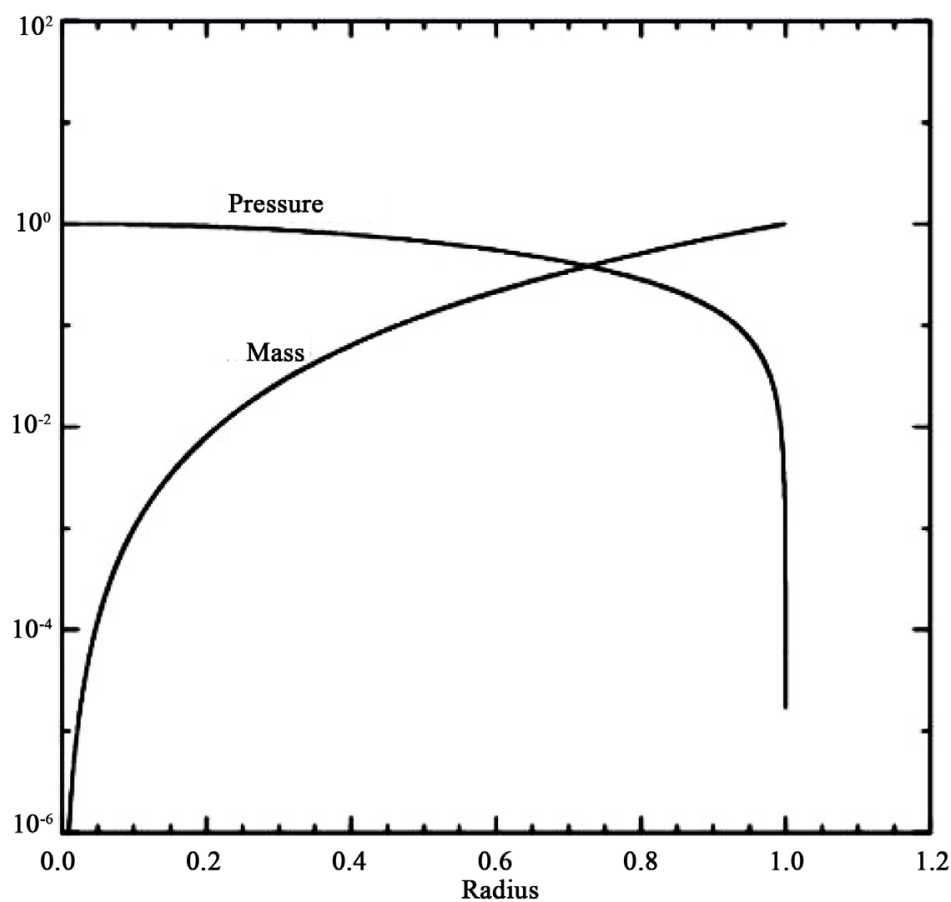

(a)

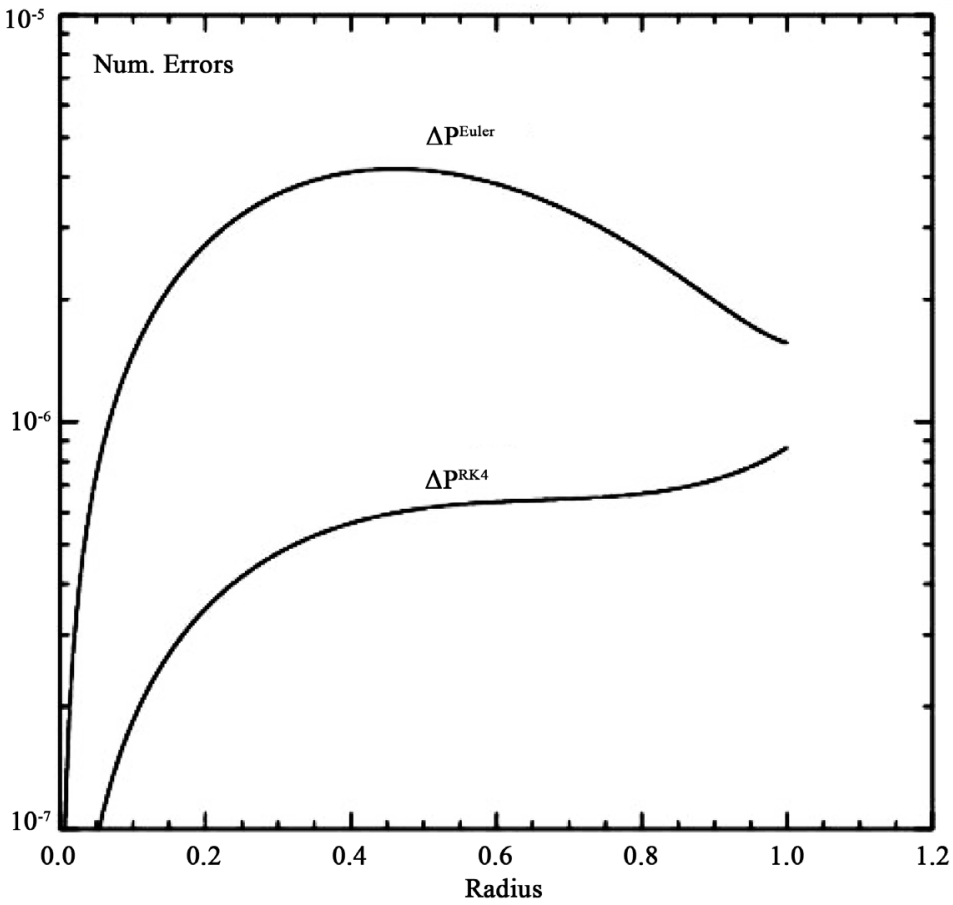

(b)

Figure 6. For test purposes the profiles of the pressure and enclosed mass of a NS that have been obtained by solving the TOV equation, using a polytropic EOS $\left(P=\mathcal{K} \rho^{\gamma}\right)$. In the lower Panel the the numerical errors for two different numerical integration methods are shown: the first order Euler and the fourth order Runga-Kutta methods using $10^{4}$ grid points compared to a reference solution that has been obtained using $10^{7}$ grid points. While the accuracy of Ruga-Kutte method is highly superior over Eulers, the effect of errors on the solutions presented here can be safely neglected. 


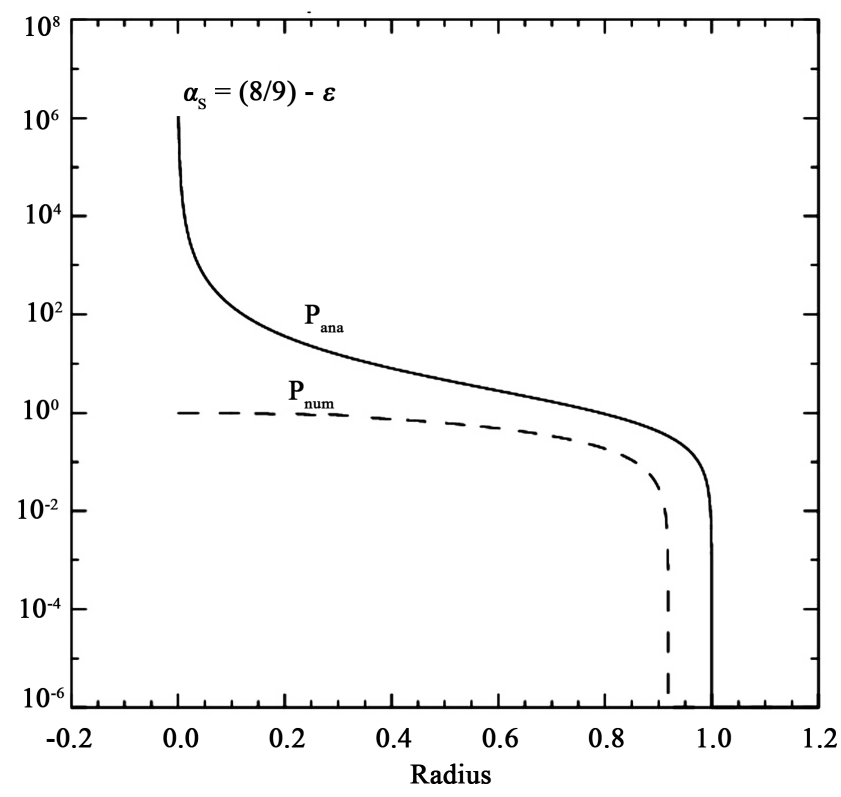

Figure 7. The analytical solution of the TOV equation $\left(P_{\text {ana }}\right)$, assuming the fluid to be incompressible (i.e., the internal energy density $\mathcal{E}_{\text {in }}=$ const $)$ for a compactness parameter $\alpha_{s}\left(\doteq R_{S} / R_{\star}\right)$ near the critical value $8 / 9$. Obviously, the solution diverges as the center is approached, where the matter becomes ultrabaric. The second profile $\left(P_{n u m}\right)$ corresponds to a numerical integration of the TOV equation using $\mathcal{E}_{\text {in }}=$ const $)$ and assuming $P(r=0)=\mathcal{E}_{\text {in }}$.
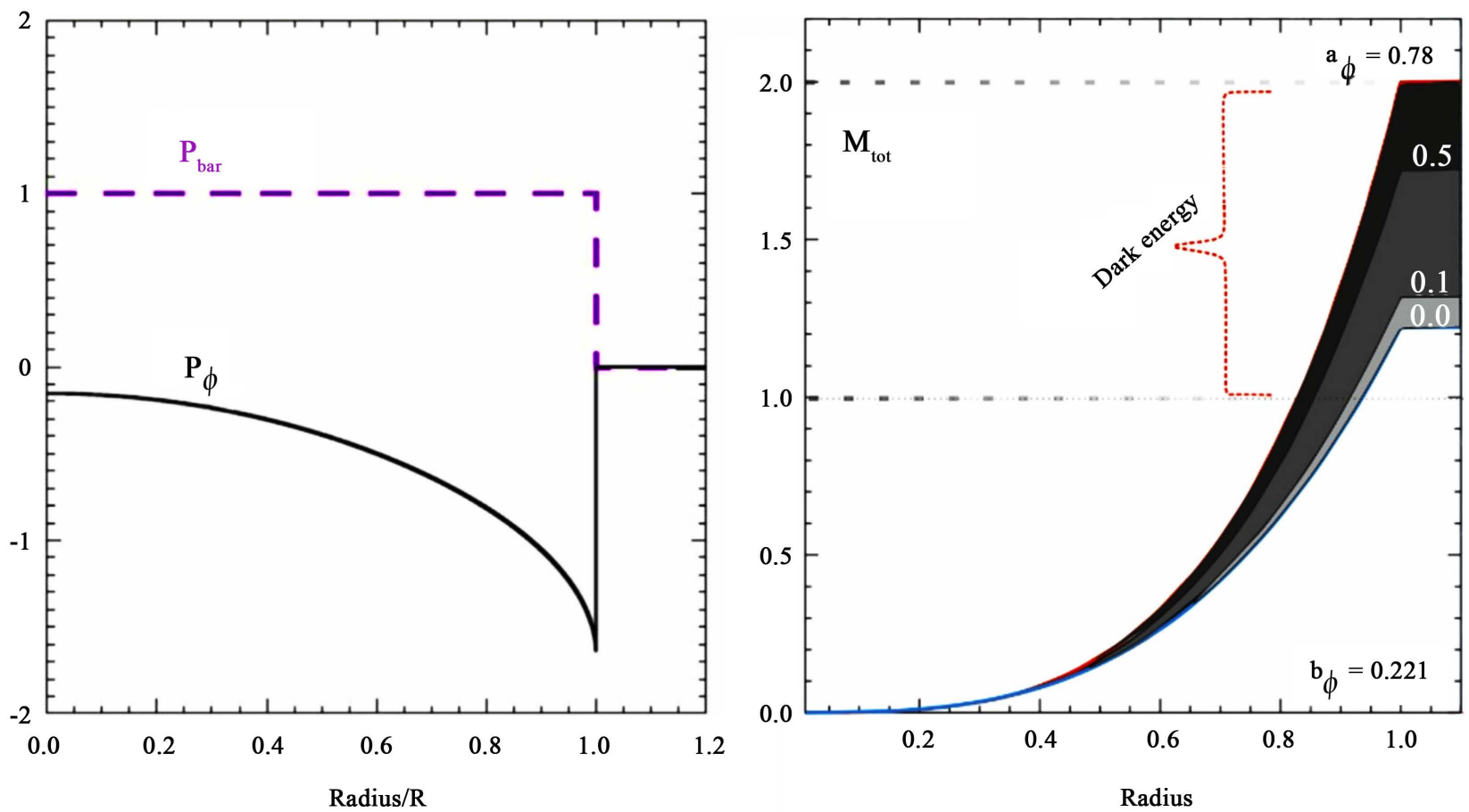

Figure 8. The radial distributions of the baryonic pressure $\left(P_{b a r}\right)$ and negative pressure $\left(P_{\phi}\right)$ inside an incompressible GQ-superfluid core (left). The enclosed mass of the baryonic matter and the gradual mass-enhancement due to dark energy is shown for different values of $a_{\phi}$ (right). 


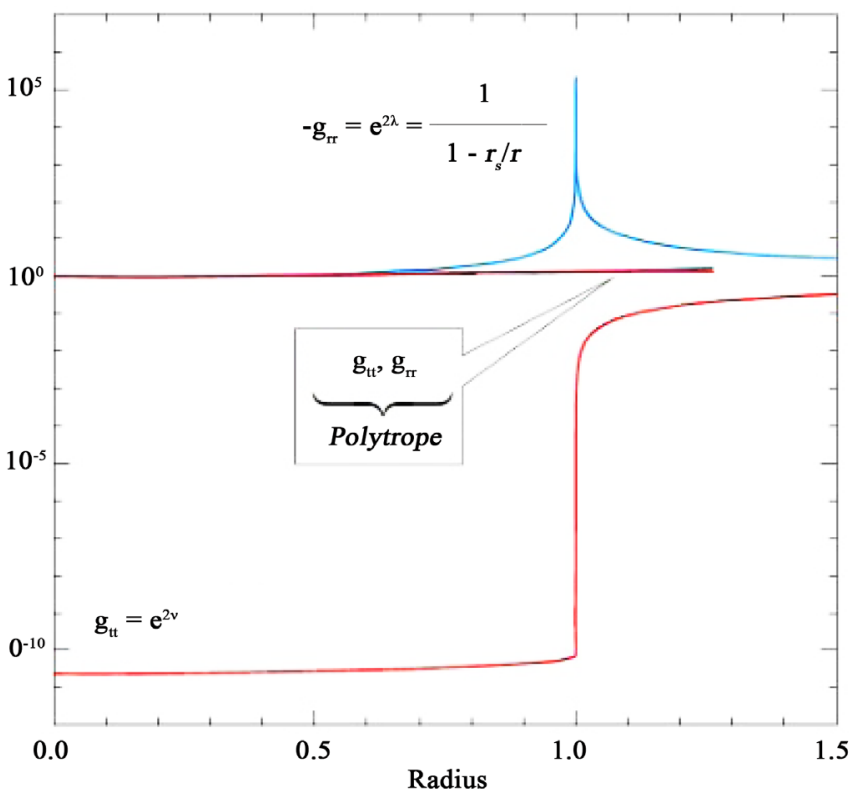

(a)

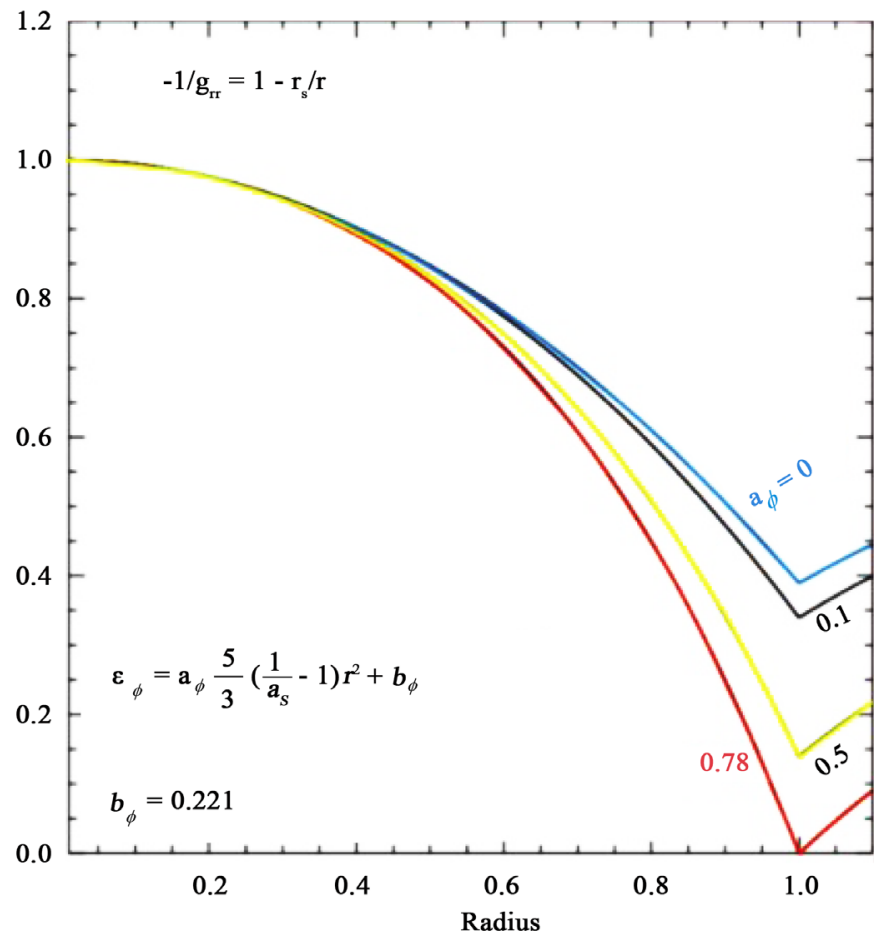

(b)

Figure 9. In the top panel we show the radial distributions of the metric coefficients $g_{r r}$ and $g_{t t}$ inside a normal NS $\left(P_{L}=\mathcal{K} \rho^{\gamma}\right.$ and $\left.P^{\phi}=0\right)$ and inside an SuSu-object $\left(P_{\text {local }}=\right.$ const and $\left.P^{\phi}=-V_{\phi}\right)$. Obviously, normal models of NSs have larger radii and considerably less compact than their SuSu-counterparts, which can be inferred from the very limited spacial variations of $g_{r r}$ and $g_{t t}$. In the lower panel, the compactness of a typical SuSu-object, expressed in terms of $-1 / g_{r r}$ is shown for different values of $a_{\phi}$. The object turns invisible if $V_{\phi}$ is calculated with $a_{\phi}=0.78$ and $b_{\phi}=0.22$. 
pressure, the enclosed effective mass (i.e. the energy due to baryon and energy enhancement by the scalar field $\phi$ ) and the inverse of the metric coefficient $g_{r r}$ for different values of the dark energy coefficient $a_{\phi}$ (see Equation (4)).

Obviously, assuming the object to have $\alpha_{S}=1 / 2$ initially, its final compactness must then increase with increasing $a_{\phi}$, i.e. with increasing the rate of dark energy injection. Using the limiting values $a_{\phi}=0.78$ and $b_{\phi}=0.22$, the scalar field is capable of injecting the energy required to deeply sink the object into spacetime and turn it invisible, where $\alpha_{S}^{c r}=1-\epsilon$, hence attaining its maximum possible value. Note that $\epsilon$ must be extremely small, but still finite in order to prevent the collapse of the object into a $\mathrm{BH}$.

These values of $a_{\phi}$ and $b_{\phi}$ yield a final effective mass that is twice as the initial baryonic mass.

Once the object has completely metamorphosed into a stellar-size SB and its compactness attained the critical limit $\alpha_{S}^{\text {cr }}$, then the spatial variation of the metric coefficient $g_{r r}$ across its surface becomes nearly singular (Figure 9), which implies that all sorts of surface radiation will be extra-ordinary redshifted, hence the object becomes observationally indifferent from its BH-counterpart. On the other hand, the metric coefficient $g_{t t}$, which is a monotonic function of the gravitational potential, appears to be fairly flat inside the object, but it undergoes a dramatic change across the surface to finally attains its weak-field values outside the object.

In addition, we have solved the TOV equation using two different EOSs: an EOS that corresponds to an incompressible GQ-superfluid inside a gradually growing SB together with a polytropic EOS for the ambient medium at different evolutionary epochs (Figure 10). While the scalar field here is set to inject energy into the SB and therefore increasing its effective mass, the surrounding medium appears to stably and comfortably adjust to the new condition of the SB.

In Figure 11 we display the Mass of fully-developed SuSu-objects and their progenitors versus $n_{c r}$ superimposed on the locations of NSs as reported by [21]. Most remarkable here is that Hulse-Taylor type pulsars are able to form SBs at their centers at much lower central density than usually required for a phase transition into quark fluids, whilst still end up twice as massive as their initial mass.

\section{Summary \& Discussion}

The here-presented model is motivated by the following issues:

- Why neither NSs nor BHs have ever been observed in the mass-range $2-5 M_{\odot}$.

- Most sophisticated EOS used to model the internal structure of NSs are based on central densities that are far beyond the nuclear density: an unknown density regime with great uncertainty.

- What is the origin of glitches observed in pulsars and young neutron stars 

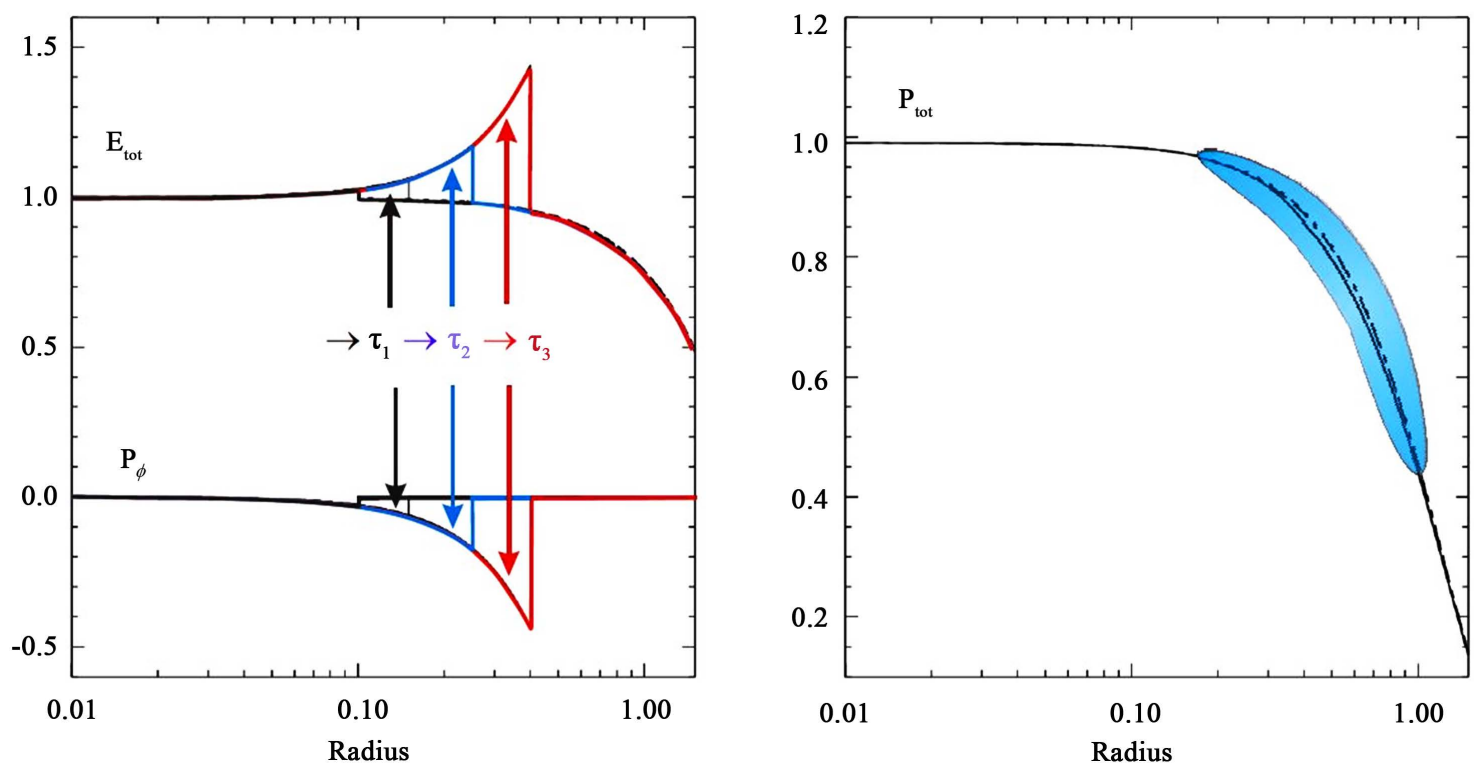

Figure 10. The profiles of the total energy density $E_{\text {tot }}$, the pressure $P_{\phi}$ induced by $\phi$ and the combined pressure $P_{\text {tot }}$ versus radius are shown for different evolutionary epochs $\tau_{1}<\tau_{2}<\tau_{3}$. Inside $r_{f}: P_{L}=0$ and $P^{\phi}=-V_{\phi}$, whereas outside $r_{f}: P_{L}=\mathcal{K} \rho^{\gamma}$ and $P_{\phi}=0$. In each epoch, the object has an SB-core overlayed by a shell of normal compressible matter obeying a polytropic EOS. Obviously, the object appears to stably and comfortably adjust itself to the mass-redistribution inside $r_{f}$, where the matter is in incompressible GQ-superfluid state.

and whether these carry information that may disclose the internal structure of their cores?

- Could massive NSs end as maximally compact dark objects, i.e. as BH-candidates?

- How does the state of matter in pulsars and NSs evolve on the cosmological time scale and whether they have hidden connection to dark matter and dark energy in cosmology?

In this respect a scenario has been presented, which can be summarized as follows:

1) Pulsars are born with embryos at their centers (here termed super-baryons -SBs). The interiors of SBs are made of GQ-superfluids and governed by the local EOS $P=\mathcal{E}=a_{\infty} n_{\text {crit }}^{2}=$ const : a purely P-incompressible superfluid state.

As a consequence, there is a universal maximum density $\rho_{q}^{\text {uni }}\left(=\mathcal{O}\left(\rho_{c r}\right)\right)$, where the momentum transfer between quarks saturates, and the coupling constant $\alpha_{\text {asym }}$ attains its universal minimum, where quarks are moving freely.

Recalling that the spatial variation of the coefficient $g_{r r}$ of the Schwarzschild metric on the nuclear length scales is negligibly small $\left(\mathrm{d} g_{r r} / \mathrm{d} l \ll 10^{-19}\right)$, the compressibility of the GQ-superfields saturates and therefore does not accept stratification by gravitational fields [24].

2) In the presence of a universal scalar field $\phi$ at the background of supranuclear densities, the injected dark energy by $\phi$ is capable of provoking a phase transition from compressible dissipative neutron fluids into incompressible GQ-superfluids. The effect of both the gravitational and scalar fields is mainly to 


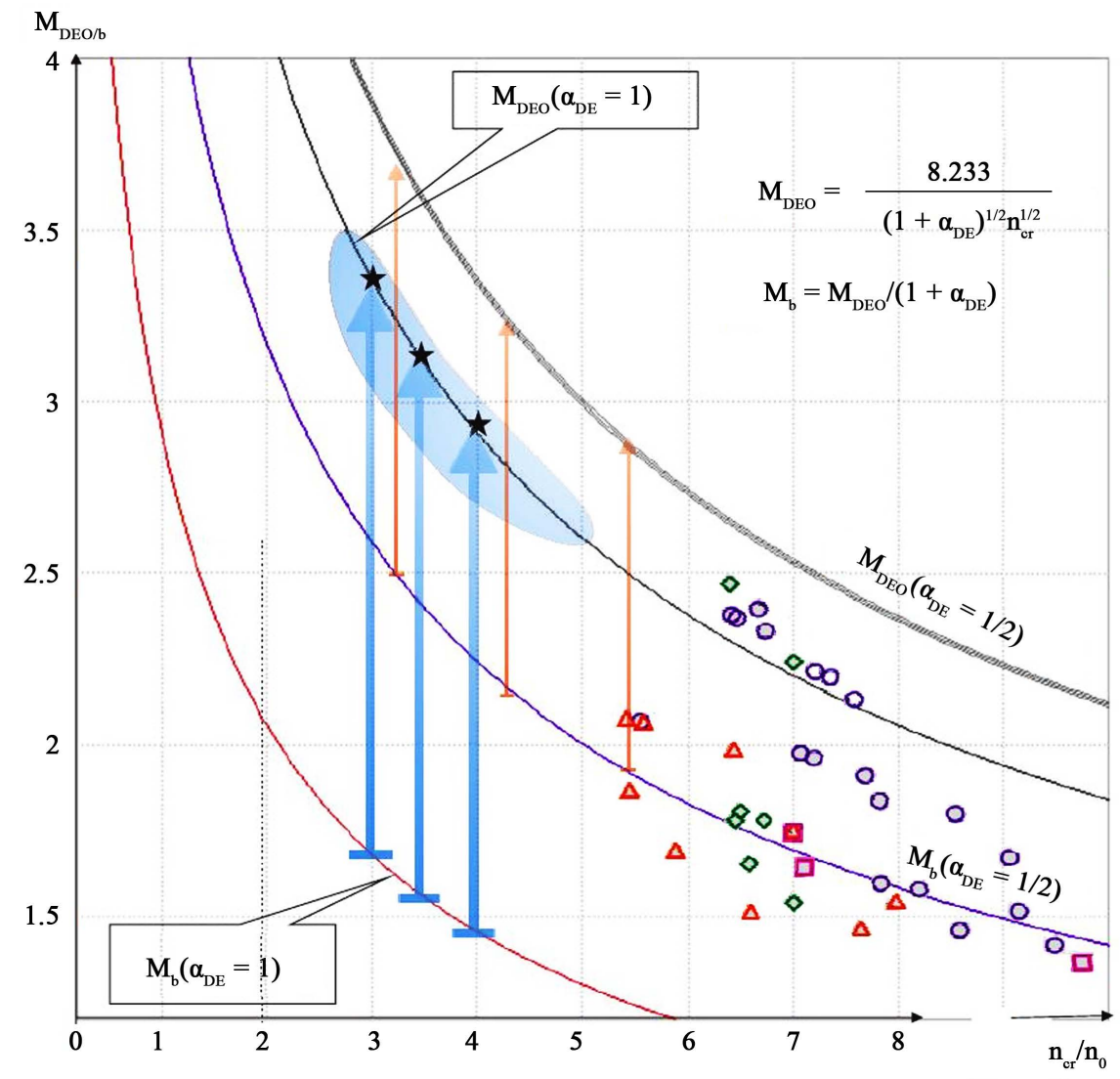

Figure 11. Upper mass limit of SuSu-objects versus critical density $n_{c r}$ (in units of $n_{0}$ ) is shown. The $\phi$-baryon interaction is set to occur at $n_{c r}$, which in turn provokes the phase transition into the incompressible GQ-superfluid state. The most probable mass-regime for SuSu-objects is marked here in blue colour. Accordingly, the progenitor of a SuSu-object with $3.36 M_{\odot}$ should be a pulsar/NS of $1.68 M_{\odot}$, provided it has an initial compactness $\alpha_{S}=1 /\left(1+\alpha_{D E}\right)=1 / 2$ and $n_{c r}=3 n_{0}$. Similarly, a Hulse-Taylor type pulsar would end as an SuSu-object of $2.91 M_{\odot}$, if its initial compactness was $\alpha_{S}=1 / 2$ and if $n_{c r}=4 n_{0}$. On the other hand, moderate and massive NSs with initial compactness $\alpha_{S} \geq 2 / 3$, i.e., $\alpha_{D E} \leq 1 / 3$, need less dark energy to become invisible SuSu-objects, though an unreasonably high $n_{c r}$ is required for triggering $\phi$-matter interaction. NSs falling in this category are to be compared with the colored small cycles and triangles, which show the approximate locations of various NS-models as depicted in Figure 11 of [21].

enhance and convert the residual strong force between nucleons into a strong force that holds quarks together [25]. This action is termed here as a "gluonization" procedure, which is equivalent to energy injection into the system, thereby maximally enhancing the effective mass of the object and turning it invisible.

It turns out that using an interaction potential of the type $V_{\phi}=a_{\phi} r^{2}+b_{\phi}$ appears to be most appropriate for maximizing the compactness of the object without significantly changing its dimensions (Figure 12).

3) In a recent study, I have shown that the glitch phenomena observed to associate the evolution of pulsars and NSs are in perfect-agreement with the 


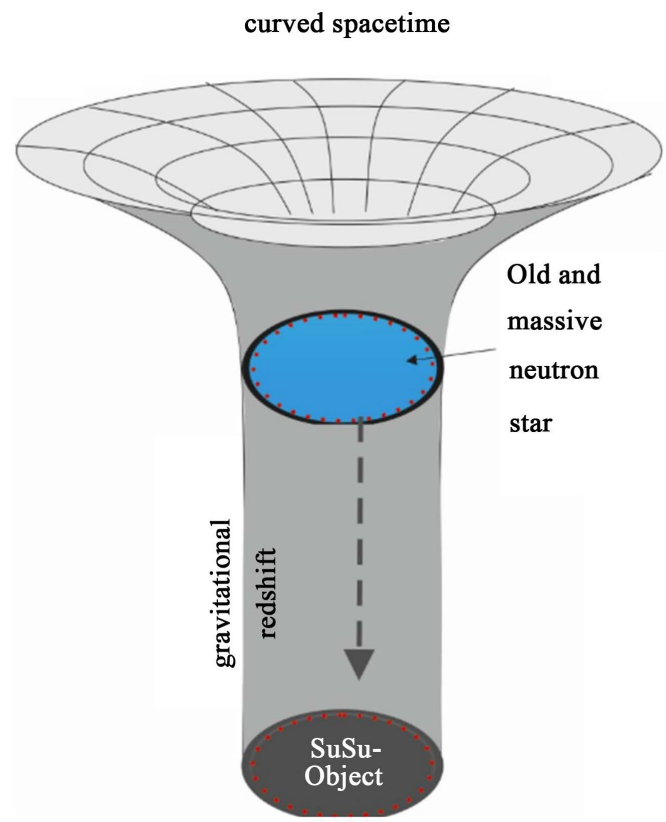

Figure 12. A schematic description of an invisible SuSu-object trapped in spacetime. The spacetime inside a SuSu-object is fairly flat, but extra-ordinary curved and nearly singular at the surface.

formation scenario of embryonic SBs at their centers.

4) Once the entire object has metamorphosed into a stellar-size SB, the spacetime in its interior would be fairly flat, but become exceedingly curved across its surface (Figure 12). Hence SBs are practically trapped in spacetime, extra-ordinary redshifted and therefore completely invisible.

5) According to the here-presented scenario, all visible pulsars and NSs must contain SBs. The gravitational significance of these SBs depends strongly on their evolutionary phase and in particular on their ages and initial compactness. Accordingly, pulsars and young NSs should be less massive than old ones, and the very old NSs should turn invisible by now.

To quantify the mass-enhancement by $\phi$, let $M_{b}$ be the mass of the NS at its birth and $M_{\phi}$ being the mass enhancement due to $\phi$. Requiring $R_{\star}>R_{S}$, then the following inequality holds:

$$
\left(1+\alpha_{D E}\right) \leq\left(\frac{3 \rho_{c r}}{32 \pi}\right)^{1 / 3} \frac{c^{2}}{G M_{b}^{2 / 3}},
$$

or equivalently,

$$
1 \leq \frac{E_{\text {tot }}}{E_{b}} \leq 2.06 \frac{\rho_{15}^{1 / 3}}{M_{b / 1.44}^{2 / 3}},
$$

where $\alpha_{D E} \doteq M_{\phi} / M_{b} . E_{\text {tot }}, \rho_{15}, M_{1.44}$ denote the total energy, the density in units of $10^{15} \mathrm{~g} / \mathrm{cc}$ and the baryonic mass of the NS in units of $1.44 M_{\odot}$, respectively.

Assuming NSs to be born with $E_{\text {tot }}=E_{b}$, then by interacting with $\phi$, they 
become more massive, hence more compact to finally reach $R_{\star}=R_{S}+\epsilon$ at the end of their luminous phase $(\epsilon \ll 1)$, which would last for approximately $10^{9} \mathrm{yr}$ or even less, depending on their initial compactness Figure 12.

Similar to atomic nuclei, I conjecture that the enormous surface stress confining the sea of GQ-superfluid inside stellar-size SBs render their surfaces impenetrable by external low energy particles, hence maintaining the eternal stability and invisibility of SuSu-objects (Figure 12). This implies that the core of a fully-developed SB would be shielded by a protecting repulsive barrier. However, due to the deep gravitational wells of SBs, such incidents would be practically observable, though they are ruled out by observations completely. Even if there were no repulsive barriers, we expect SBs to still be stable against mass-enhancement from external sources. Let a certain amount of baryonic matter, $\delta \mathcal{M}_{b}$, be added to the object via accretion from external sources. Then the relative increase of $R_{\star}$ compared to $R_{S}$ scales as: $\frac{\delta R_{\star}}{\delta R_{S}} \simeq \frac{\rho_{c r}}{\tilde{\rho}_{\text {new }}}$, where $\tilde{\rho}_{\text {new }}$ is the average density of the newly settled matter. Unless $\tilde{\rho}_{\text {new }} \geq \rho_{\text {cr }}$, which is forbidden under normal astrophysical conditions, the SuSu-object would react stably. However, in the case of super-Eddington accretion or merger, the newly settled matter must first decelerate, compressed and subsequently becomes virially hot, giving rise therefore to $\tilde{\rho}_{\text {new }} \ll \rho_{c r}$. On the other hand, such events would lower the confinement stress at the surface and would turn the quantum jump of the energy density at $R_{\star}$, which falls abruptly from approximately $\mathcal{E} \approx 10^{36} \mathrm{erg} / \mathrm{cc}$ at $R_{\star}$ down to zero outside it, into an extra-ordinary steep pressure gradient in the continuum. While such actions would smooth the strong curvature of spacetime across $R_{\star}$, they would enable SuSu-objects to eject quark matter into space with ultra-relativistic speeds, which is forbidden. Nonetheless, even if this would occur instantly, then the corresponding time scale $\tau_{d}$ would be of order $\Lambda_{j} / c$, where $\Lambda_{j}$ is the jump width in centimeters. Relating $\Lambda_{j}$ to the average spacing between two arbitrary particles $\left(\sim n^{-1 / 3}\right)$, this yields $\tau_{d} \approx 10^{-24} \mathrm{~s}$, which is many orders of magnitude shorter than any known thermal relaxation time scale between arbitrary luminous particles.

Although electromagnetic activities and jets have never been observed in dark matter halos, they are typical events for systems containing black holes. Recalling that supermasive GBECs are dynamically unstable [26], our results here address the following two possibilities:

- If the onset of $\phi$-baryon interaction indeed occurs at $n_{c r}$, then the majority of the first generation of stars must have collapsed into UCOs and subsequently became SuSu-objects, rather than collapsing into stellar $\mathrm{BHs}$ with $M \leq 5 \times M_{\odot}$. These objects are expected to conglomerate into dark matter halos over several big bang events and to embed the galaxies in the observable universe [27]. This conclusion is in line with recent observations of NASA's Spitzer Space Telescope, which reveal the existence of primordial 
galaxies, such as GN-z11, whose age might be even bigger than that of our universe [28].

- The passivity of DM to electromagnetic radiation may indicate that the collective effect of the repulsive forces of a cluster of SuSu-objects is repulsive on smaller length scales and attractive on the larger ones. Hence approaching luminous matter will be forced to deviate from face-to-face collisions with the cluster of SuSu-objects, though n-body and SPH-numerical calculations are needed here to verify this argument.

\section{References}

[1] Baym, G. (1995) Nuclear Physics A, 590, 233. https://doi.org/10.1016/0375-9474(95)00238-V

[2] Link, B. (2012) MNRAS, 422, 1640-1647. https://doi.org/10.1111/j.1365-2966.2012.20740.x

[3] Baranghi, C. (2008) Physica D, 237, 2195. https://doi.org/10.1016/j.physd.2008.01.010

[4] Baggaley, A.W. and Laurie, J. (2014) Physical Review B, 89, Article ID: 014504. https://doi.org/10.1103/PhysRevB.89.014504

[5] Dix, O.M. and Zieve, R.J. (2014) Physical Review B, 90, Article ID: 144511.

[6] Espinoza, C.M., Lyne, A.G., Stappers, B.W. and Kramer, C. (2011) MNRAS, 414, 1679. https://doi.org/10.1111/j.1365-2966.2011.18503.x

[7] Haensel, P., Potekhin, A.Y. and Yakovlev, D.G. (2007) Neutron Stars 1. Springer, Berlin. https://doi.org/10.1007/978-0-387-47301-7

[8] Baym, G. and Chin, S.A. (1976) Physics Letters B, 62, 241-244. https://doi.org/10.1016/0370-2693(76)90517-7

[9] Hampel, M., Fischer, T., Schaffner-Bielich, J. and Liebendörfer, M. (2012) APJ, 748, 70. https://doi.org/10.1088/0004-637X/748/1/70

[10] Camenzind, M. (2007) Compact Objects in Astrophysics. Springer, Berlin.

[11] Chapline, G. and Nauenberg, M. (1977) Physical Review D, 16, 450. https://doi.org/10.1103/PhysRevD.16.450

[12] Kislinger, M.B. and Merley, P.D. (1978) Astrphysical Journal, 219, 1017-1028. https://doi.org/10.1086/155866

[13] Hujeirat, A.A. and Thielemann, F.-K. (2009) MNRAS, 400, 903. https://doi.org/10.1111/j.1365-2966.2009.15498.x

[14] Shuryak, E. (2017) Reviews of Modern Physics, 89, Article ID: 035001.

[15] Stachel, J. (2017) Private Communications.

[16] Hujeirat, A.A. (2017) Cornell University Library.

[17] Eya, I.O. and Urama, J.O. (2014) International Journal of Astrophysics and Space Science, 2, 16. https://doi.org/10.11648/j.ijass.20140202.11

[18] Eya, I.O., Urama, J.O. and Chukwude, A.E. (2017) APJ, 840, 56. https://doi.org/10.3847/1538-4357/aa6b55

[19] Serim, M.M., Sahiner, S., Cerri-Serim, D., et al. (2017) MNRAS, 471, 4982. https://doi.org/10.1093/mnras/stx1771

[20] Glendenning, N. (2007) Special and General Relativity. Springer, Berlin. https://doi.org/10.1007/978-0-387-47109-9 
[21] Lattimer, J.M. and Prakash, M. (2011) From Nuclei to Stars: Festschrift in Honor of Gerald E. Brown. World Scientific Publishing, Singapore.

[22] Naoyuki Sakumichi, N. and Suganuma, H. (2015) Physical Review D, 92, Article ID: 034511. https://doi.org/10.1103/PhysRevD.92.034511

[23] Bethke, S. (2007) Progress in Particle and Nuclear Physics, 58, 351-386.

[24] Hujeirat, A.A. (2018) Journal of Modern Physics, 9, 51-69. https://doi.org/10.4236/jmp.2018.91004

[25] LHCb Collaboration (2015) Physical Review Letters, 115, Article ID: 072001.

[26] Hujeirat, A.A. (2012) $M N R A S, 423,2893$.

[27] Hujeirat, A.A. (2018) Journal of Modern Physics, 9, 70-83. https://doi.org/10.4236/jmp.2018.91005

[28] Castellano, M., Amorin, R., et al. (2016) A\&A, 590, A31. 